\title{
Simultaneous Catalytic Conversion of Acid Pretreated Biomass into High-quality Syngas and Bio-oil at Mild Temperature
}

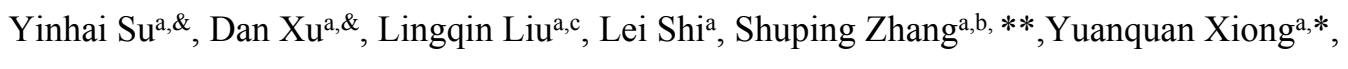
Huiyan Zhanga

${ }^{a}$ Key Laboratory of Energy Thermal Conversion and Control of Ministry of Education, School of Energy and Environment, Southeast University, Nanjing 210096, China

${ }^{\mathrm{b}}$ School of Energy and Power Engineering, Nanjing University of Science and Technology, Nanjing 210094, China

${ }^{\mathrm{c}}$ Center for Biorefining and Department of Bioproducts and Biosystems Engineering, University of Minnesota, 1390 Eckles Ave., St. Paul, MN, 55108, USA

\& These authors contributed equally to this work and should be considered as co-first authors

*Corresponding Author: E-mail: yqxiong@seu.edu.cn (Xiong Y.).

**Corresponding Author: E-mail: zhangpeyton@163.com (Zhang S.). 
Tab. S1 Relative content of main chemical species in bio-oil obtained from raw and pretreated rice husk samples.

\begin{tabular}{|c|c|c|c|c|c|c|c|}
\hline & RH-N & RH & PTRH60 & PTRH80 & $\begin{array}{c}\text { PTRH10 } \\
\text { 0 }\end{array}$ & $\begin{array}{c}\text { PTRH12 } \\
\text { 0 }\end{array}$ & $\begin{array}{c}\text { PTRH14 } \\
\text { 0 }\end{array}$ \\
\hline Acids & 20.27 & 2.12 & 0.98 & 1.12 & 1.82 & 1.07 & 0.95 \\
\hline Acetic acid & 17.55 & 2.12 & 0.98 & 1.12 & 1.82 & 1.07 & 0.95 \\
\hline Propanoic acid & 1.86 & -- & -- & -- & -- & -- & -- \\
\hline $\begin{array}{c}\text { Butanoic acid, } \\
\text { 4-hydroxy- }\end{array}$ & 0.86 & -- & -- & -- & -- & -- & -- \\
\hline Ketones & 16.51 & -- & -- & -- & -- & -- & -- \\
\hline $\begin{array}{l}\text { 2-Propanone, } \\
\text { 1-hydroxy- }\end{array}$ & 6.37 & -- & -- & -- & -- & -- & -- \\
\hline 1-Hydroxy-2-butanone & 1.23 & -- & -- & -- & -- & -- & -- \\
\hline 2-Cyclopenten-1-one & 1.49 & -- & -- & -- & -- & -- & -- \\
\hline $\begin{array}{l}\text { 2-Cyclopenten-1-one, } \\
\text { 2-methyl- }\end{array}$ & 1.19 & -- & -- & -- & -- & -- & -- \\
\hline $\begin{array}{l}\text { 2-Cyclopenten-1-one, } \\
\text { 2-hydroxy- }\end{array}$ & 2.93 & -- & -- & -- & -- & -- & -- \\
\hline $\begin{array}{c}\text { 1,2-Cyclopentanedione, } \\
\text { 3-methyl- }\end{array}$ & 2.33 & -- & -- & -- & -- & -- & -- \\
\hline Levoglucosenone & 0.97 & -- & -- & -- & -- & -- & -- \\
\hline
\end{tabular}

\begin{tabular}{cccccccc}
\hline Aldehydes & $\mathbf{4 . 8 1}$ & -- & -- & -- & -- & -- & -- \\
\hline Succindialdehyde & 1.74 & -- & -- & -- & -- & -- & - \\
Pentanal & 3.07 & -- & -- & -- & -- & -- & -- \\
\hline
\end{tabular}

\begin{tabular}{|c|c|c|c|c|c|c|c|}
\hline Furans & 15.92 & -- & -- & -- & -- & -- & -- \\
\hline Furfural & 2.97 & -- & -- & -- & -- & -- & -- \\
\hline 2-Furanmethanol & 2.79 & -- & -- & -- & -- & -- & -- \\
\hline $2(5 \mathrm{H})$-Furanone & 1.97 & -- & -- & -- & -- & -- & -- \\
\hline $\begin{array}{l}\text { Benzofuran, } \\
\text { 2,3-dihydro- }\end{array}$ & 6.61 & -- & -- & -- & -- & -- & -- \\
\hline $\begin{array}{l}\text { 5-Hydroxymethylfurfur } \\
\text { al }\end{array}$ & 0.79 & -- & -- & -- & -- & -- & -- \\
\hline $\begin{array}{l}\text { 5-Hydroxymethyldihyd } \\
\text { rofuran-2-one }\end{array}$ & 0.79 & -- & -- & -- & -- & -- & -- \\
\hline
\end{tabular}

\begin{tabular}{cccccccc}
\hline Phenols & $\mathbf{3 3 . 9 2}$ & $\mathbf{8 6 . 0 9}$ & $\mathbf{8 6 . 0 9}$ & $\mathbf{8 6 . 7}$ & $\mathbf{8 8 . 7 2}$ & $\mathbf{8 7 . 5 6}$ & $\mathbf{8 6 . 7}$ \\
\hline $\begin{array}{c}\text { Benzaldehyde, } \\
\text { 2-hydroxy- } \\
\text { Phenol }\end{array}$ & 0.86 & -- & -- & -- & -- & -- & -- \\
& 3.88 & 66.44 & 66.57 & 67.43 & 67.14 & 63.06 & 56.71
\end{tabular}




\begin{tabular}{|c|c|c|c|c|c|c|c|}
\hline Phenol, 2-methoxy- & 1.39 & -- & -- & -- & -- & -- & -- \\
\hline Phenol, 2-methyl- & 2.12 & 5.6 & 4.39 & 4.28 & 5.22 & 5.65 & 5.92 \\
\hline Phenol, 2,5-dimethyl- & 1.30 & -- & -- & -- & -- & -- & 1.96 \\
\hline p-Cresol & 2.35 & -- & -- & -- & -- & -- & -- \\
\hline Phenol, 3-methyl- & 1.86 & 14.07 & 15.13 & 14.99 & 16.36 & 18.85 & 19.47 \\
\hline Phenol, 2,4-dimethyl- & 1.70 & -- & -- & -- & -- & -- & -- \\
\hline Phenol, 4-ethyl- & 3.65 & -- & -- & -- & -- & -- & 2.64 \\
\hline $\begin{array}{c}\text { Phenol, } \\
\text { 4-ethyl-2-methyl- }\end{array}$ & 1.15 & -- & -- & -- & -- & -- & -- \\
\hline $\begin{array}{l}\text { 2-Methoxy-4-vinylphen } \\
\text { ol }\end{array}$ & 1.49 & -- & -- & -- & -- & -- & -- \\
\hline Catechol & 4.17 & -- & -- & -- & -- & -- & -- \\
\hline Phenol, 4-(2-propenyl)- & 1.12 & -- & -- & -- & -- & -- & -- \\
\hline Phenol, 4-(2-propenyl)- & 1.48 & -- & -- & -- & -- & -- & -- \\
\hline $\begin{array}{l}\text { 1,2-Benzenediol, } \\
\text { 4-methyl- }\end{array}$ & 2.43 & -- & -- & -- & -- & -- & -- \\
\hline Hydroquinone & 1.33 & -- & -- & -- & -- & -- & -- \\
\hline $\begin{array}{l}\text { 1,4-Benzenediol, } \\
\text { 2-methyl- }\end{array}$ & 0.82 & -- & -- & -- & -- & -- & -- \\
\hline 4-Ethylcatechol & 0.81 & -- & -- & -- & -- & -- & -- \\
\hline Esters & 5.79 & -- & -- & -- & -- & -- & -- \\
\hline $\begin{array}{l}\text { 1,2-Ethanediol, } \\
\text { monoacetate }\end{array}$ & 1.5 & -- & -- & -- & -- & -- & -- \\
\hline $\begin{array}{c}\text { Propanoic acid, 2-oxo-, } \\
\text { methyl ester }\end{array}$ & 1.53 & -- & -- & -- & -- & -- & -- \\
\hline $\begin{array}{l}\text { 2-Propanone, } \\
\text { 1-(acetyloxy)- }\end{array}$ & 1.29 & -- & -- & -- & -- & -- & -- \\
\hline $\begin{array}{l}\text { 10-Undecenoic acid, } \\
\text { octyl ester }\end{array}$ & 1.47 & -- & -- & -- & -- & -- & -- \\
\hline Others & 2.78 & 3.71 & 7.30 & 2.00 & -- & -- & 0.93 \\
\hline Ethane, 1,1-diethoxy- & -- & 3.71 & -- & -- & -- & -- & -- \\
\hline $\begin{array}{c}\text { Hydroquinone, } 2 \mathrm{TMS} \\
\text { derivative }\end{array}$ & 0.8 & -- & -- & -- & -- & -- & -- \\
\hline Phenol, & & & & & & & \\
\hline $\begin{array}{c}\text { 3-methyl-4-(methylthio } \\
\text { )- }\end{array}$ & 0.99 & -- & -- & -- & -- & -- & -- \\
\hline Prednisolone Acetate & 0.99 & -- & 7.3 & -- & -- & -- & -- \\
\hline Diisooctyl phthalate & -- & -- & -- & 0.9 & -- & -- & -- \\
\hline Benzenemethanol, & & & & & & & \\
\hline $\begin{array}{c}\text { 2-[bis(4-hydroxyphenyl } \\
\text { )methyl]- }\end{array}$ & -- & -- & -- & 1.1 & -- & -- & -- \\
\hline Benzoic acid, & -- & -- & -- & -- & -- & -- & 0.93 \\
\hline
\end{tabular}


5-[2-chloro-4-(trifluoro

methyl)phenoxy]-2-nitr

o-, ethyl ester

1-Leucine,

N-ethoxycarbonyl-N-m

3.71

ethyl-, octadecyl ester

\begin{tabular}{cccccccc}
\hline PAHs & $\mathbf{0}$ & $\mathbf{8 . 0 8}$ & $\mathbf{5 . 6 3}$ & $\mathbf{1 0 . 1 8}$ & $\mathbf{9 . 4 6}$ & $\mathbf{1 1 . 3 7}$ & $\mathbf{1 1 . 4 1}$ \\
\hline Naphthalene & -- & 3.22 & 3.13 & 3.38 & 3.54 & 4.45 & 3.98 \\
Naphthalene, 2-methyl- & -- & 1.79 & 1.66 & 1.89 & 1.88 & 2.13 & 2.3 \\
Naphthalene, 1-methyl- & -- & 1.05 & 0.84 & 1.65 & 0.95 & 1.11 & 1.15 \\
Benzocycloheptatriene & -- & -- & -- & 0.89 & 1.29 & -- & -- \\
Biphenyl & -- & -- & -- & 0.82 & 0.91 & 1.51 & 1.54 \\
Dibenzofuran & -- & 1.03 & -- & 1.03 & -- & 1.05 & 1.23 \\
Fluorene & -- & 0.99 & -- & 0.52 & 0.89 & 1.12 & 1.21 \\
\hline
\end{tabular}


Fig.S1 Calibration Curve of Phenol.

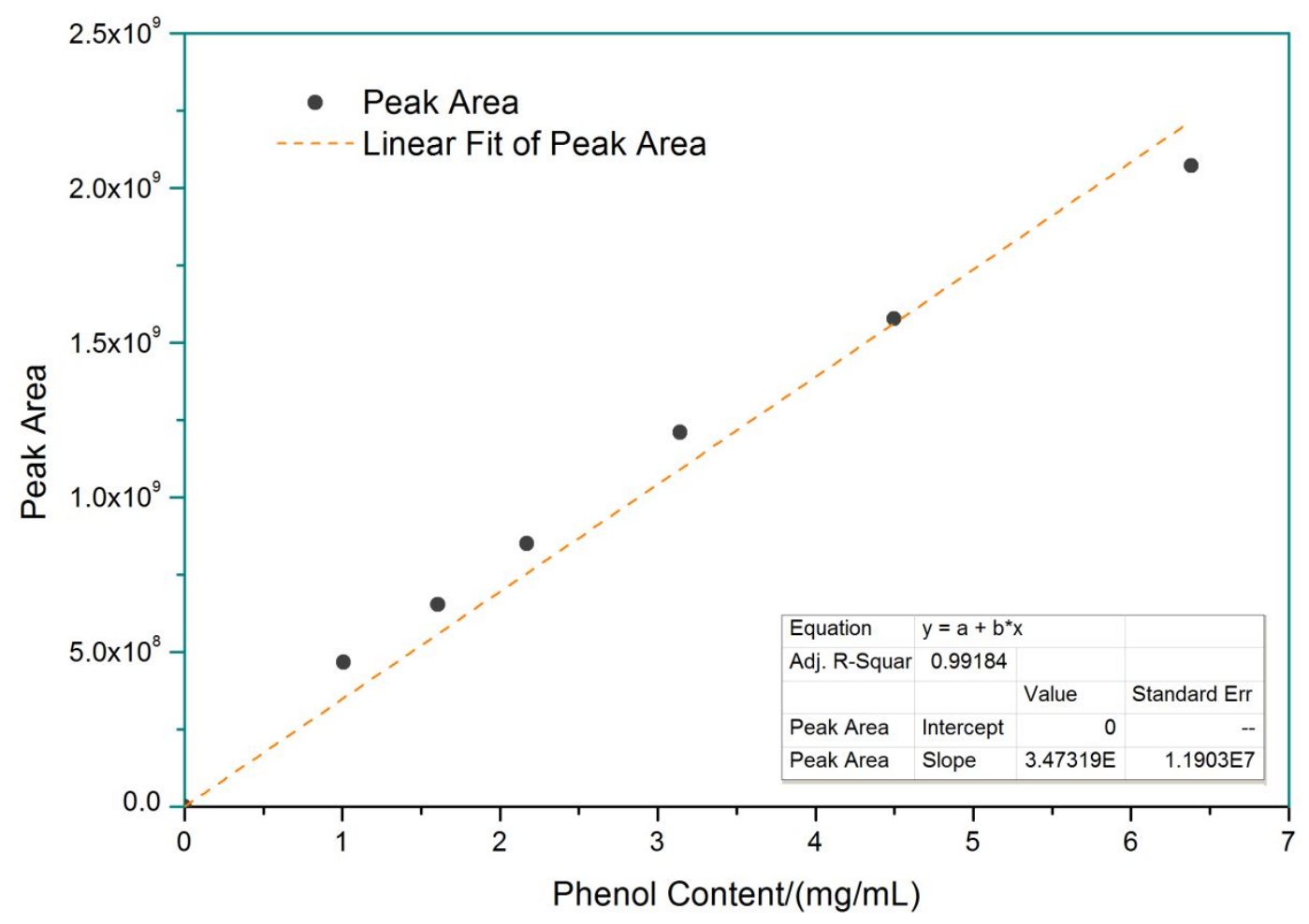

